\title{
"Why The Scale Goes Up Again?" A Socio-Ecological Exploration of Weight Loss Challenges among Female University Students
}

\author{
Ayat Ibrahim \\ Dep. of Edu. Psychology and Counseling, \\ Kulliyyah of Education, \\ International Islamic University Malaysia, \\ Kuala Lumpur, Malaysia \\ rabbani.writer@gmail.com
}

\author{
Haniza Rais \\ Dep. of Edu. Psychology and Counseling, \\ Kulliyyah of Education, \\ International Islamic University Malaysia, \\ Kuala Lumpur, Malaysia \\ hanrais@iium.edu.my
}

\begin{abstract}
The purpose of this study was to explore the weight management and weight maintenance challenges experienced by obese female university students through a support group that acted as an intervention programme. The study is a phenomenological research involving four female students of a public university in Malaysia who were having problems with weight loss. They were selected with three inclusion criteria: female, had a body mass index of $\geq 30 \mathrm{kgm}^{-2}$ and was currently seeking or had previously tried a method of losing weight. Interview protocols were used to guide interaction in the support group, and data were collected from regular focus group discussions occurring in the support group, journal writing, and products of the support group activities. Verbatims were transcribed and "meaning units" were derived from significant overlapping ideas. Using the socio-ecological model, the challenges of weight management among the participants were identified to exist at the individual, interpersonal and community levels. Of these three, the individual level was noted to have the most prominent number of challenge factors caused by poor self-control and the participants' psychological states. Various issues related to poor self-control were the most frequently occurring themes among the participants who wanted to lose weight. In view of the findings, counsellors, dietitians or healthcare providers need to consider counselling techniques that can empower clients to manage their thoughts and emotions effectively as this will increase their self-efficacy in selfcontrol.
\end{abstract}

Keywords: Weight loss, weight management, challenges in sustainable weight loss, poor selfcontrol, sabotaging thoughts, socio-ecological model

\section{INTRODUCTION}

Globally, overweight and obesity are issues that are expanding in current healthcare. Modern lifestyle that includes the consumption of fast food and processed food, and being sedentary contributes to this epidemic. Annual costs for obesity are estimated around $\$ 1.1$ billion, $€ 5.1$ billion and \$1 billion for the US, Germany and Canada, respectively (Tremmel et al., 2017). Malaysia was ranked the first among Southeast Asian countries with high adult obesity rate $(\mathrm{Ng}$ et al., 2014). The prevalence of overweight and obesity in Malaysia was $48.3 \%$ and $48.6 \%$ for men and women in the year 2013 ( $\mathrm{Ng}$ et al., 2014). In 1996, the prevalence of adult obesity in Malaysia was only $4.4 \%$ of the population. 
But within 20 years, this percentage has increased drastically. In 2015, the national prevalence of obesity was $17.7 \%$ of the population as reported by the Malaysian National Health and Morbidity Survey (Ministry of Health, 2015). In a very recent national health and morbidity survey, one in every two Malaysian adults is found to be overweight or obese (Institute for Public Health, 2020). Despite the numerous efforts made, it is still unclear how far Malaysia's healthcare interventions have succeeded in curbing and combating obesity among the Malaysian public. While weight loss is often desired among obese individuals, only a handful of these individuals managed to sustain their weight loss over time.

Over the past two decades, clinical interventions have proven effective in establishing initial weight loss. However, a majority of those who completed a treatment for obesity regained the weight they had lost after five years following the treatment (Jeffery et al., 2000). A review of research on weight loss management revealed that while 10-15\% weight loss can be expected on average in behaviorally-based weight loss treatment programmes, four years later, most of this weight is usually regained, with individuals typically sustaining around $4 \%$ loss from the initial weight (Jeffery et al., 2000).

\section{LITERATURE REVIEW}

\section{Definition and Concepts of Weight Loss Challenges}

Challenges in weight management refer to anything or anyone that hinders the practice of healthy behaviors, such as healthy eating or being physically active. Overweight is weight that is $10 \%$ to $20 \%$ above the standard or "ideal" body weight for individuals of the same age, sex and height (Lagua \& Claudio, 2004). The World Health Organization (WHO) officially defines obesity as a body mass index (BMI) of $30.0 \mathrm{kgm}^{-2}$ and greater. Thus, weight loss challenges in this study refer to barriers faced by overweight or obese people in their effort to lose weight.

Prevalence research revealed that Malaysians are getting fatter (i.e., overweight and obese) and rounder (i.e., abdominal obesity) through the years from 2009-2015. According to a recent article by Lum (2018), Malaysia is Asia's fattest country where it was documented that approximately half of Malaysia's population are overweight or obese. In addition, females were found to be more predominant in obesity and abdominal obesity as compared to males in Malaysia (Tan et al., 2019). This suggests that effective intervention promoting sustainable weight loss is warranted. The challenges in losing weight are influenced by several issues at differing levels of the socio-ecological model. At the individual level, the list of barriers to weight management and sustainability includes low nutrition knowledge and skills (Ali, Baynouna \& Bernsen, 2010), low motivation (Al-Mohaimeed \& Elmannan, 2017; Metzgar et al., 2014; Abolhassani et al., 2012; Befort et al., 2008) and health problems (Abolhassani et al., 2012; Metzgar et al., 2014; Ali et al., 2010). At the interpersonal level, the biggest challenges are lack of family support (Al-Mohaimeed \& Elmannan, 2017; Abolhassani et al., 2012; Metzgar et al., 2014) and competing demands, especially lacking the time for child care (Ali et $a l ., 2010)$. At the next level, i.e., the institutional or organizational level, the lack of exercise facilities (Ali et al., 2010), unhealthy eating during social gatherings (Al-Mohaimeed \& Elmannan, 2017), and lack of support from the workplace (Abolhassani et al., 2012; Befort et 
al., 2008) are contributing factors to weight management problems. At the community level, socio-cultural norms or environmental pressures may affect those who plan to lose weight (Ali et al., 2010; Metzgar et al., 2014). Lastly, at the policy and systems level, street safety concerns are issues that hinder the citizens of a nation from practicing and perpetuating a culture of maintaining good health through physical exercises (Ali et al., 2010).

Pursuant to the importance of maintaining a healthy body weight, the study seeks to shed light on the struggles of losing weight among females in Malaysia. It answered the main research question posed, i.e., "What are the challenges faced by obese female students who attend weight management support groups?" The findings will instil a basic understanding about the experience of losing weight, particularly in dealing with the obstacles faced, in addition to helping to suggest possible ways of overcoming the obstacles. By exploring the perceived barriers or difficulties faced by the participants, it is hoped that counsellors or dietitians will be able to identify areas to be improved in providing the services of weight management to interested individuals who are fighting off obesity.

\section{METHODOLOGY}

\section{Research Design}

This study is a qualitative study of a phenomenological nature. A support group as the setting of the study was created among adult participants to share their weight management experiences in the group. Phenomenology focuses specifically on the subjective experiences of individuals. The researcher observed the interaction and dialogues among the participants and their world, thereby allowing her to address important questions pertaining to their experiences in sustaining weight loss. Thus, in this case, the phenomena of interest are the challenges of losing weight among selected obese individuals.

\section{Participants}

The participants were four female university students, aged between 20 and 25 years, selected from the International Islamic University Malaysia via purposive sampling. Since this study adopted the phenomenological approach that needed to uncover female struggles in losing weight and maintaining a weight loss, specific selection criteria were exercised to ensure that the participants selected had roughly experienced the same issues, and could provide information related to the same phenomenon. The selection or inclusion criteria were (i) female, (ii) with a body mass index of $\geq 30 \mathrm{kgm}^{-2}$; and (iii) at the time of data collection, was seeking or had previously tried a method of losing weight. Four participants who met the inclusion criteria and consented to participate in the study were recruited. 


\section{Setting}

A support group for weight management was the setting for the study. A weight loss support group programme, which offered weekly group counselling sessions, was conducted throughout the month of October in 2015. The sessions used a cognitive behavioral therapy and equipped the participants with the skills of cognitive restructuring, i.e., the act and process of identifying negative or destructive thought patterns that contributed to their weight management issues, and replacing them with positive or constructive thoughts that would help them to overcome the problems of sustaining their weight loss. While providing counselling sessions to the participants, it also acted as a focus group for data collection in which discussions about weight problems, weight loss techniques and sustainability challenges were laid out on the table. The researcher was the instrument for collecting the data as she led the focus group discussion by playing the role of the team leader. The sessions were set in campus and were held during the long-semester session.

\section{Instruments}

Interview protocols were created based on the research question. The techniques of group interviewing used a set of questionnaires consisting of primarily open-ended questions and some close-ended questions. A group proposal to weight management was created and vetted by a qualified counselor and dietetics experts. Data were collected from focus group discussions, personal intake forms, journal writing and the products of support group activities (See Table 1).

Table 1:

Instruments, Data Collection Methods and Expected Data

\begin{tabular}{|c|c|c|c|c|c|}
\hline Objective & Research Question & & Interview Protocol & $\begin{array}{c}\text { Methods of } \\
\text { Collecting Data }\end{array}$ & Expected Data \\
\hline $\begin{array}{l}\text { To identify the } \\
\text { sources of } \\
\text { challenges among } \\
\text { female university } \\
\text { students that } \\
\text { contributed to } \\
\text { barriers in their } \\
\text { process of losing } \\
\text { weight. }\end{array}$ & $\begin{array}{l}\text { What are the } \\
\text { challenges faced } \\
\text { by obese female } \\
\text { students who } \\
\text { attended a weight } \\
\text { management } \\
\text { support group? }\end{array}$ & $\begin{array}{l}\text { 1) } \\
\text { 2) } \\
\text { 3) } \\
\text { 4) }\end{array}$ & $\begin{array}{l}\text { What challenges do } \\
\text { you face while trying } \\
\text { to lose weight? } \\
\text { Can you describe the } \\
\text { difficulties you face? } \\
\text { What is the hardest } \\
\text { part of losing weight? } \\
\text { What makes your } \\
\text { effort fail? }\end{array}$ & $\begin{array}{l}\text { 1) Personal intake } \\
\text { forms } \\
\text { 2) Focus group } \\
\text { discussion in } \\
\text { "Support } \\
\text { group" }\end{array}$ & $\begin{array}{l}\text { 1) } \begin{array}{l}\text { Sources of } \\
\text { challenges }\end{array} \\
\text { 2) } \\
\text { Reasons for } \\
\text { failing to lose } \\
\text { weight. }\end{array}$ \\
\hline
\end{tabular}

\section{Data Analysis and Trustworthiness}

The data collected were transcribed as verbatim. To elicit the essence of the participants' responses, the researcher looked for overlapping or recurring ideas that were of significance to the study's objectives and research questions. These statements were then grouped into identified main ideas before being generated into themes. The inductive process of narrowing the main ideas into themes explained the central phenomenon under investigation. 
Triangulation of the data was established through referential adequacy and member checking. The findings and interpretations about weight loss challenges among overweight or obese were checked against previous literature and current research such as the works of Al-Mohaimeed and Elmannan (2017), Metzgar et al. (2014), and others. As for the member checking procedure, the interview protocols were checked by two lecturers in the field of counseling. After analysing the FGD data, the participants were asked to review the accuracy of the researcher's interpretations and descriptions of their experiences. For this purpose, regular consultations with the participants were made. At the same time, to prevent biases from the researcher's own experiences as dietitian, the researcher continuously discussed her personal reactions with her supervisors, and reviewed her reflective journals to minimise interference, such as the researcher's own tendencies to make assumptions or generalizations based on the participants' habits or behaviour.

\section{FINDINGS}

\section{Participant Information}

Eight participants responded to the advertisement. Interested participants either called or sent text messages to the researcher to learn more about the weight loss support group programme. After the screening process, all of them met the selection criteria. However, during the prescreening session, only four were able to attend the first meeting as scheduled. A detailed profile of all participants is given in Table 2 .

Table 2:

Profile of the Participants

\begin{tabular}{lcccc}
\hline \multicolumn{1}{c}{ Data } & \multicolumn{4}{c}{ Participant's Details } \\
\hline Name Initial & AW & NA & ND & PF \\
Age & 25 & 20 & 21 & 21 \\
Year of Study & 4 & 1 & 1 & 2 \\
$\begin{array}{l}\text { Initial Weight } \\
\text { (as of 2 }\end{array}$ & $91 \mathrm{~kg}$ Oct 2015) & $82 \mathrm{~kg}$ & $81 \mathrm{~kg}$ & $90 \mathrm{~kg}$ \\
Body Mass Index (BMI) & $33.8 \mathrm{kgm}^{-2}$ & $32.9 \mathrm{kgm}^{-2}$ & $35.1 \mathrm{kgm}^{-2}$ & $30.8 \mathrm{kgm}^{-2}$ \\
\hline
\end{tabular}

The participants were aged between 21-25 years and were in Year 1, Year 2 and Year 4 of their study at the IIUM. They had BMIs ranging between 30 and $35 \mathrm{kgm}^{-2}$, which indicated that they were all in the BMI range of being obese.

Two of the participants were actively involved in sports. AW (a pseudo name) had been playing handball since the past three years of the study's data collection, and represented the university in tournaments. She played this sport mainly out of interest and passion. Also physically active, PF (a pseudo name) had been exercising in the gymnasium with the aim of losing weight. 
Meal replacement or supplement had been followed by AW and NA (pseudo names) as a method of losing weight, while the other two participants had tried being on a controlled and balanced meal plan to cut down their calorie intake.

All of them were very dissatisfied with their current weight and viewed their efforts as a failure. Wanting a healthier and happier life was the driving factor behind the weight loss efforts of three of the participants (i.e., NA, ND and PF), while the fourth participant, AW, was very much concerned with being able to play her favourite sport safely and comfortably. All of the participants planned to reduce between 20 and 50\% of their current weight. However, their targeted weight reduction was not in compliance with the recommended Ministry of Health guideline which suggests just a $10 \%$ weight loss over a 6-month therapy period (MOH, 2004).

\section{Challenges to Weight Management}

Based on the socio-ecological model (Stokols, 1996), three categories emerged as the major underlying challenges for these participants. They can be categorized into three levels, namely the individual level, interpersonal level and community level. The three levels are illustrated in Figure 1. Under each level, there are emerging themes and subthemes that explain the challenges in greater detail.

\section{Individual Level}

With reference to the socio-ecological model (Gregson et al., 2001), individual-level challenges involved a person's knowledge, attitudes, beliefs and personality traits related to certain behavioural, psychological and cognitive factors. At the individual level, two major themes of weight management challenges emerged, which were (i) poor self-control and eating habits, and (ii) psychological factors.

\section{Poor Self-Control and Eating Habits}

Poor self-control led to high energy intake and low energy expenditure. This, in turn, kept the weighing scale on the increase. The specific problems of self-control that the participants experienced were consuming big portions in every meal, taking high-calorie and low-nutrient food, having frequent meal times beyond the regular, munching on food out of meal times, and having sabotaging thoughts.

\section{Consuming Big-Portioned Meals}

The habit of consuming big portions of a meal was very difficult for obese female students to discard. Even research has documented that the difficulty in controlling hefty appetites was a barrier to weight loss among women (Ali et al., 2010). Thus, when they were given knowledge and skills in monitoring portion control, the participants were better able to control their food intake (Metzgar et al., 2014):

\footnotetext{
"I was like, eating chicken and rice a lot. If I eat, I can even finish up 2 pieces of chicken." [AW]
} 
"I'm just like her. If I eat, I'll ensure, there is rice. I want a lot of rice. Then, I want to have vegetables, fish and chicken. My plate must look full." [ND]

Eating large meals can be associated with fast eating, a habit that hinders a person from realizing that they were already full before overeating (Beck, 2008).

"I think it's because I'm rushing. There is no other time to eat so I'll eat quickly. I hardly feel full when I eat." [AW]

\section{Consuming High-Calorie, Low-Nutrient Food}

The consumption of high-calorie, low-nutrient food, or more easily termed as "junk food," was common among obese female students. Junk food includes processed meat, sweetened beverages, sweets, and crackers. Desire for good tasting food is marked as one of the top three challenges among participants of weight management programmes (Call et al., 2019). Two of the participants disclosed that they were struggling with this issue. One of the contributing factors was the attitude of resistance, i.e., resisting to overcome desires or cravings.

"The problem is, when I want to buy fruits there, I'll find crackers instead (laughing)." [PF]

"If in UIA, I like to have 'CikguDrink'. Still it's unhealthy - rich in sugar." [ND]

Another cause that led to their wight management problem was poor problem-solving ability. Instead of identifying junk food as a main contributor of their weight management problem, it was used as a "problem solver" to other issues, such as preventing themselves from being sleepy in class.

"It was sweet. Whenever I go to class, I'll bring along sweets because I always feel sleepy." [ND]

\section{Frequent Meal Times Beyond the Regular Routine}

Proper meal times are typically referred to as breakfast, lunch and dinner. For one of the participants, eating was completely compulsory, something that could not be sacrificed for anything at all. Thus, she would always have a number of fixed meal times that she religiously observed in a day. Unfortunately, however, opportunities to eat more than the planned meals often cropped up, thereby increasing her food intake that undermined her weight loss effort. This contributed to the intake of more additional energy daily.

"I will usually eat according to my time. For example, in the afternoon, we have Zuhr break right, so I'll eat during that time. Or, if the day is full with classes, I will eat at 10-11 am. Then, my next meal would be at $5 \mathrm{pm}$. However, later on, at night, there will probably another one. Like after 5 pm, there will be another mealtime." [AW] 
In another study, women perceived that having planned meals everyday within the recommended energy intake was important to avoid overeating (Metzgar et al., 2014). Without planning ahead, they felt as if they were not prepared to face "food challenges."

\section{Consistently Munching on Finger Food Outside the Planned Meal Times}

The desire to munch on finger food throughout the day provided zero nutrients with a lot of excess energy intake per day. Two of the obese female students were facing this problem. The underlying issue was related to the self-gratification mechanism of an individual. Eating was viewed as a way to make people feel comfortable and at ease. Hence, it became one of the favorite pastimes of an individual. This habit was prevalent, especially when obese female students were not engaged in any useful activities.

“When I do nothing, I'll eat." [AW]

"If I'm busy, I cannot be bothered with eating. But when I'm free, I was like... Let's eat!" $[\mathrm{AW}]$

\section{Sabotaging Thoughts}

The attitude of always giving in to sabotaging thoughts affected the obese female students' eating behaviours.

"If I see a lot of rice, I'll say to myself--It's ok. 'I'll eat lesser tomorrow.' Then when tomorrow comes, I'll say, "Oh no, why did I eat a lot. [But] Never mind, I'm hungry. " [ND]

All of the participants believed that exercising plays a significant role in losing weight. However, two of them found it difficult to engage in physical activities due to laziness and the feelings of discomfort and shame.

"It's so hard to exercise because... at first, because I'm lazy. That is the biggest factor when I'm fat. Then, when I was about to jog, I feel discomfort with all the fats shaking with me. I'm ashamed. When I want to run, I'll also feel heavy. ” [NA]

"I'm so lazy to do exercise because I feel heavy. All of the excessive fats will be shaken then I'll feel ashamed." [ND]

On top of that, some self-sabotaging thoughts made their way into the minds of the participants, thereby increasing their already poor self-control and breaking down all desires and intention to engage in a physical exercise. The participants admitted to creating excuses not to engage in any exercise as a result of having these negative thoughts, reasoning with themselves that they had to spare their energy for other important tasks:

"Then, if I want to exercise, I'll create excuses though I actually have the time. But I'll make it like: I have a lot of work now; I have to save my energy for other tasks, too (instead of spending the time for exercise)." [NA] 
"That day, I planned to exercise during weekends. When it comes, I said to myself.

Never mind, I haven't cleaned up the room yet." [ND].

On the contrary, another study found that factors related to participants' saying they did not have time for physical exercises and finding no enjoyment in the physical activities were the reasons for obese people not to engage in the activities (Metzgar et al., 2014)

\section{Psychological Factors}

The second theme that emerged under the individual level category was psychological factors. These include the issue of low motivation, believing that healthy food is expensive and always feeling hungry.

\section{Low Motivation}

Extreme ways of losing weight that are actually not sustainable may kill one's motivation to lose weight. This is especially true when the success of losing a few kilograms of weight seemed worthless with the regaining of the previous kilograms after a while. The issue of low motivation was consistent with the findings of barriers to weight management among Arab women (Ali et al., 2010). Regaining the lost weight after a short span of time was especially demotivating when sustainable weight loss was not achieved.

"Two years ago, I lost a lot of weight but regained it all back later on. Only within 1 year, all my effort seemed worthless." [AW].

The poor outcome of previous attempts had de-motivated obese female students, thereby creating a negative belief system regarding the success of weight management.

"After that, whatever effort I gave--seemed not working." [AW].

Another participant also felt de-motivated when her struggle with dieting failed to result in any success. This was disclosed in the participant's intake form, written by NA during the first session of the support group.

"The hardest part of losing weight is controlling the consumption of food but the weight remained still." [NA]

Peer influence and a low readiness to change can also be the factors detracting the motivation of obese individuals to persist in their weight loss effort (Abolhassani et al., 2012 ; Metzgar et al., 2014).

\section{Belief that Healthy Food Is Expensive}

Many tend to believe that healthy food is expensive. Such a belief is also prevalent among obese individuals. The belief tends to prevent them from actively searching and buying healthy food as part of their weight loss intervention. Two of the participants appeared to have this 
misperception when they mentioned the difficulty of getting fruits and vegetables on campus due to their price:

"If you want to eat fruits every day, we have them at HS. They sell cut fruits, under the stairs. But they are expensive you know, one ringgit each." [AW]

A previous study, i.e., Russell et al. (2013), reported the higher costs of fresh, healthy food, as compared to the lower prices of processed foods, had been cited by obese individuals to be a challenge for them in practicing a healthy lifestyle. Whether this is an incapacitating psychological state is worth researching into by counsellors and dietitians.

\section{Always Feeling Hungry}

The state of always feeling hungry had led one of the participants to binge, i.e., indulging in a frequent and excessive intake of food, which most likely had increased her calorie intake per day. She was unable to differentiate between the pangs of hunger and binging and a constant craving for food (Beck, 2008). Thus, the participants felt that binging was an urgent need that must always be fulfilled due to their feeling hungry all the time.

“Always feel hungry. Such feeling is always there." [NA]

\section{Interpersonal Level}

Interpersonal level includes all social interactions between the individual and their family, colleagues, social networks and others who gave them a social identity and role definition (Gregson et al., 2001) Some significant challenges arose when weigh loss efforts involved social interaction with family and friends. Thus, the major theme under the interpersonal level category was insufficient support from family and friends.

\section{Insufficient Support System}

\section{Friends vs Efforts to Lose Weight}

All of the participants revealed that their friends' social invitations always threw them into a dilemma. AW, for example, admitted she could not control her appetite during eat outs, especially when accompanied by friends. Social life and interaction is important in the Malaysian culture, and is always associated with eating in a social gathering.

"For example, in the evening, my friends invited me to eat out. That's the problem. I had actually planned what to eat, but when they asked me out, my entire plan went into the drain." [AW]

"For example, they invite me to go to the café. All of them are small in size. I don't have the heart to refuse." [PF]

At this point, the desire to enjoy food overrode the importance of controlling food intake. Thus, obese female students preferred to accept the invitation from friends rather than being resisted. 


\section{Finishing Up Others' Food}

The desire to eat more than needed was reinforced by the offering of food by friends. In order to prevent food wastage, the participants had the tendency to accept the leftover food offered by others who, most of the time, would eat in small amounts.

"They are not the kind of people that eat a lot. If they eat, they only do so in small portions. For example, the fried rice dinner that we used to order, they only consume a quarter of it. Then, they will give me the rest. That's why I become like this. I keep accepting and eating their [leftover] food." [NA]

\section{Family Tradition and Culture}

A common challenge found both in the family and among friends was being unable to resist food pushers. The inability to say no to food pushers could be due to the deeply rooted norms that have existed in the society, social circles or family tradition for a long time. Some families have rules that everybody in their circles must eat whatever food the mother has cooked, as it is everyone's filial duty to please the mother. In some cases, with high fatty diets permeating their eating patterns in the family, obese individuals would find it difficult to escape the excessive energy intake whenever they stayed at home. Being unassertive had prevented them from resisting the persuasiveness of food pushers in the family.

"I think, my mother would get hurt. There are times when I refused to eat then my mother responded in a very disappointing tone: You return home only for a short while but refuse to eat what I have cooked. " [PF]

This finding was consistent with another study that described family traditions acting as a facilitator of or barrier to healthy lifestyle changes (Russell et al., 2013). A family that grew up eating a lot of vegetables would lead a lifestyle of adequate fibre and minerals intake. In contrast, a family that grew up with fried dishes three times a day might expose the members to chronic illnesses.

\section{Community Level}

Community-level challenges involved social networks, norms or standards such as public agenda and media agenda (Gregson et al., 2001). At the community level, 3 themes emerged. They were availability of an abundance of food, few options of healthy food choices, and the norm of eating as a social means of celebration.

\section{Eating as a Common Norm of Social Celebration}

The culture of eating as a compulsory agendum in any gatherings or meetings made it difficult for obese female students to gain support for their weight loss efforts. Eating is a common norm for social celebrations among Malaysians, as in the case in other cultures as well. A study by 
Russell et al. (2013) described how behavioural changes that obese individuals wanted to effect were made difficult by their food-related cultural tradition. Social factors or cultural elements do present some great challenges that increase the plight of weight watchers in losing weight (Call et al., 2019)

"Especially when we do tasks with friends like attending usrah (learning circle). The trend is: food must be served. There's one time when our leader invited us to have some physical activity at Taman Layang-Layang. But then, when we arrived, we started eating. How to exercise then?" [AW]

\section{Food Abundance}

The easy availability of food in abundant amounts and varieties was a challenge to those who want to lose weight. Schools and homes do not provide as much food as can be found in the university. Female students who are obese tend to experience significant amounts of weight gain when they begin university life. As university campuses often have numerous food outlets with a huge variety of choices at affordable prices, obese female students are often led to temptations they cannot resist:

"When I was in my secondary school, my weight was only $50 \mathrm{~kg}$. When I came here in university, the weight increased drastically. " [NA]

"If I am at home, I don't eat much. We don't really cook actually. But when I'm in the university, the food is readily available." [WA]

\section{Few Options of Healthy Food}

At the same time, few options of healthy food are available in their surroundings. A participant mentioned that university cafes or canteens do not cook vegetables into healthy meals, which prevents obese individuals from practicing a healthy diet and lifestyle and staying committed to it (Russell et al., 2013). Even if they make an effort to buy fruits and vegetables for the purpose of healthy eating, there will be storage issues that cannot be solved. Where will they store the fruits and vegetables at their hostel units on campus?

"I want to buy fruits every Wednesday at the night market. Somehow, fruits cannot sustain long so I cannot buy in big amounts. Furthermore, the vegetables that the café provided were not being prepared in a healthy way. " [PF] 


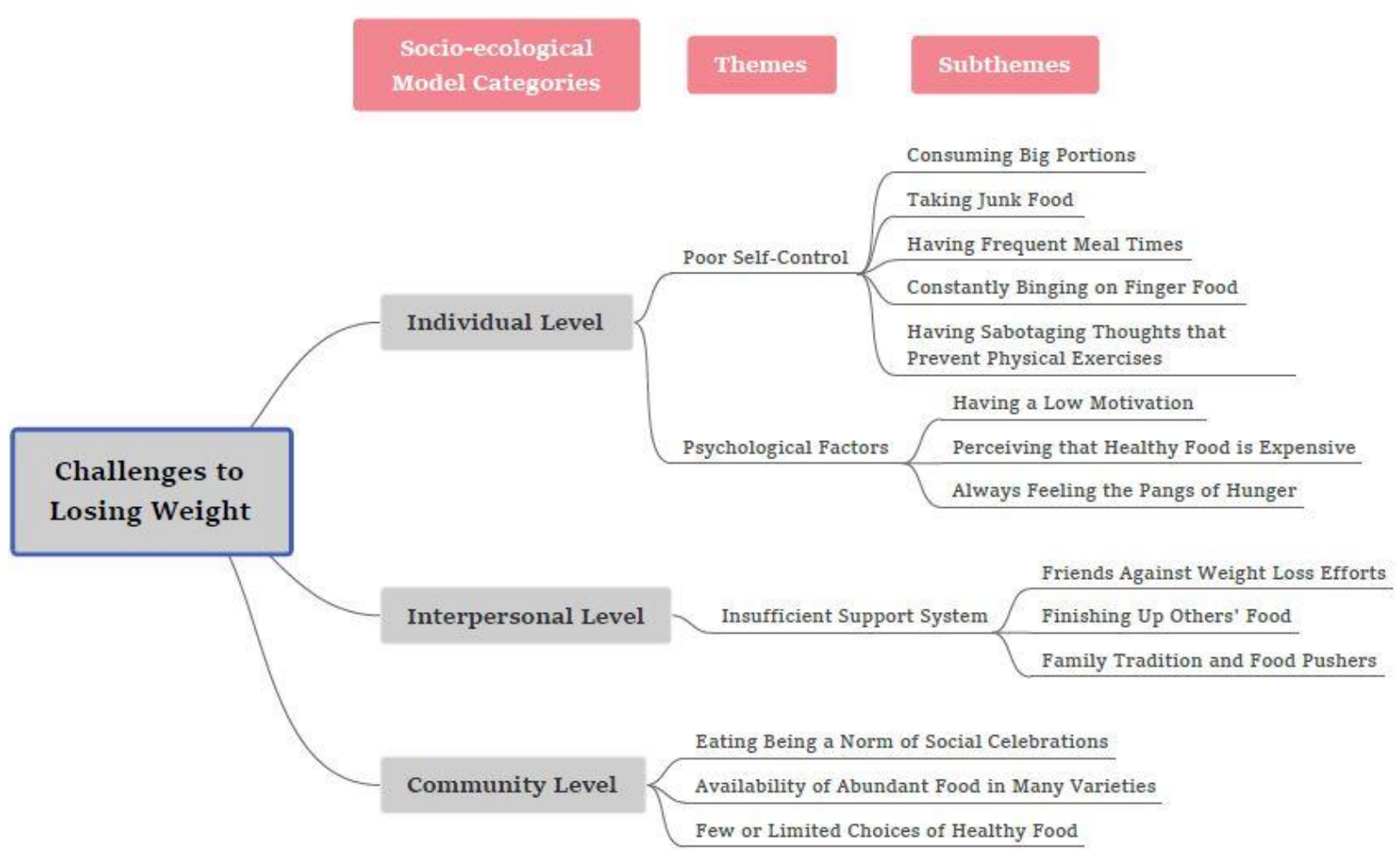

Figure 1: Challenges to Losing Weight Experienced by the Study's Participants

\section{DISCUSSION}

Issues related to poor self-control were the most frequently recurring themes among the participants who wanted to lose weight. Unhealthy habits that resulted from poor self-control included consuming big portions for a meal, high-calorie and low-nutrient food consumption, having frequent meal times, binging on finger food, and having sabotaging thoughts that impeded the intention to do physical activities. Factors contributing to poor self-control involved a person's cognitive and emotion management. Techniques from both the behavioural and cognitive therapies would be helpful in assisting obese individuals to modify their eating habits.

Low motivation as a barrier to weight management was mostly caused by the previous failure in weight loss attempts among the research participants. Thus, weight maintenance is important to ensure a continuous effort in managing weight. Part of the strategies used by successful weight maintainers over a 12-month period were cognitive restructuring, exercising, practicing assertiveness skills, and eating style changes (Miller, 1981). This is further supported by studies that reported changes in negative beliefs and self-defeating private monologues as important to the success of a 12-month weight-control programme (Teixira, 2004).

Insufficient support from friends and family members was among the concerns of obese female students. The ability to optimize the network among family and friends for information, encouragement, emotional support and enhancing the environment to support behaviour change are the elements that defined social support (Spahn et al., 2010). Dietitians or counsellors may 
assist clients in identifying potential family and community support as well as coaching them on how to effectively elicit this support (Call et al., 2019, Spahn et al., 2010). This includes developing the skills of communication, encountering sabotaging thoughts, and being assertive in a polite way (Beck, 2008). At the community level, all research participants agreed that food availability in their campus increases their food consumption as compared to when they are at home where food is not as abundant. According to Hill and Peter (1998), two environmental factors that promote overeating are food availability and portion sizes as well as high-fat diets offered, especially in fast food outlets.

\section{Educational Implications}

The first practical contribution of the present research is that it provides data for developing psycho-educational modules in the area of behavioural changes, specifically in regard to shaping a healthy eating behaviour and positive lifestyle changes among the public, and treating eating disorders among students and teenagers. Since body image is one of the primary concerns impacting individuals' self-esteem and self-confidence, counsellors in educational settings such as secondary schools, colleges and universities may gain insights from this research and use the findings to guide their practices in equipping clients with the necessary skills of weight control. The second implication of this study is drawn from the understanding of weight loss barriers which challenge the current weight management practices among obese individuals, particularly university students. None of the participants reported having a lack of knowledge about food and calories, which actually contributed to their weight status and obesity. These findings suggest that a program to educate young people about food and nutrients leading to the practice of a healthy diet and lifestyle is extremely paramount. This content should even be included in our school curriculum, at as early a stage as pre-school education. In addition, counsellors-especially those working in schools and universities--must be competent in educating and empowering clients in exercising self-control and interpersonal skills to help them manage weight issues, rather than focusing on only imparting information on calories and on the do's and don'ts for dieting to clients. 


\section{CONCLUSION}

After analysing the challenges to weight loss, questions arise pertaining to how far counsellors, dietitians or healthcare providers are concerned about clients' difficulties or barriers to further assist them in overcoming the challenges. Since most of the challenges were related to selfcontrol issues, dietitians' self-efficacy in applying counselling skills to further motivate and empower clients in modifying their behaviour, specifically their eating habits or patterns, is a critical success factor that requires further exploration.

With the limited number of dietitians against the increasing population of obese people among Malaysians, an effective support group should be instituted to optimize the impact of a weight loss intervention. Further research using experimental or intervention designs may help to recognize the therapeutic effects of having a support group while optimizing the energy and time of the intervention.

Funding: The writers would like to thank IIUM for the internal grant, RIGS16-144-0308 (Project Title: Nutrition Counseling: Using Support Group to Explore Weight Management Challenges Among Female Youth) which allowed us to undertake the study and make the publication of this work possible. 


\section{REFERENCES}

Abolhassani, S., Irani, M.D., Sarrafzadegan, N., Raiei, K., Shahrokhi, S., Poumoghaddas, Z., Mohammadifard, N., Roohafza, H., Asgary, S. Moattar, F. (2012). Barriers and facilitators of weight management in overweight and obese people: Qualitative findings of ABASSOM project. Iranian Journal of Nursing and Midwifery Research,17, (3) $205-210$.

Al-Mohaimeed, A. A., \& Elmannan, A. A. A. (2017). Experiences of barriers and motivators to weight-loss among Saudi people with overweight or obesity in Qassim Region-A qualitative study. Open access Macedonian journal of medical sciences, 5(7), 1028.

Ali, H.I., Baynouna, L.M., and Bernsen, R.M. (2010). Barriers and facilitators of weight management: perspectives of Arab women at risk for type 2 diabetes. Health and Social Care in the Community, 18 (2), 219-228.

Call, C. C., Schumacher, L. M., Rosenbaum, D. L., Convertino, A. D., Zhang, F., \& Butryn, M. L. (2019). Participant and interventionist perceptions of challenges during behavioral weight loss treatment. Journal of behavioral medicine, 42(2), 353-364.

Beck, J.S. (2008). The Beck Diet Solution: Train your brain to think like a thin person. USA: Oxmoor House Inc.

Befort, C.A., Stewart, E.E., Smith, B.K., Gibson, C.A, Sullivan, D.K., \& Donelly, J.E. (2008).Weight maintenance, behaviors and barriers among previous participants of a university-based weight control programme. International Journal of Obesity,32, 519526.

Gregson, J., Foerster, S.B., Jones, L., Benedict, J., Hersey, J., Lewis, J., Zotz. A.K. (2001).System, environmental, and policy changes: using the social-ecological model as a framework for evaluating nutrition education and social marketing programmes with low-income audiences. Journal of Nutrition Education, 33 (suppl 1), S4 - S15.

Hill, J. O. and Peters, J.C. (1998). Environmental contributions to the obesity epidemic. Science, 280 (5368), 1371-1374.

Institute for Public Health (2020). National health and morbidity survey (NHMS) 2019 : Noncommunicable diseases, healthcare demand and health literacy-key findings.

Jeffery, R. W., Drewnowski A., Epstein, L.H. Stunkard, A.J., Wilson, G.T., Wing, R.R., \& Hill, D.R. (2000). Long-term maintenance of weight loss: current status. Health Psychology, 19, 5-16.

Lagua, R.T., Claudio, V.S. (2004). Nutrition and diet therapy reference dictionary. Australia, Blackwell Publishing. 
Lum, M. (2018, Aug 14). Malaysia is asia's fattest country. The Star Online. Accessible at https://www.thestar.com.my/lifestyle/health/2018/08/14/fat-state-of-affairs/

Mahoney, M. (1991). Human change processes: The scientific foundations of psychotherapy. New York : Basic Books.

Metzgar C. J., Preston, A. G., Miller D.L. \& Nickols-Richardson, S. M. (2014). Facilitators and barriers to weight loss and weight loss maintenance: A qualitative exploration. Journal of Human Nutrition and Dietetics, 28 (6), 593-603

Miller, P. M.,Sims K. L. (1981) Evaluation and component analysis of a comprehensive weight control programme. Nutrition Journal of Obesity, 5(1), 57-65

Ministry of Health Malaysia (2004). Clinical practice guidelines on management of obesity. From http://www.acadmed.org.my/cpg/CPG-Obesity.pdf.

Ministry of Health Malaysia . (2015) National Health and Morbidity Survey 2015 (NHMS 2015). Volume II-Non- communicable diseases, risk factors \& other health problems [Internet]. Kuala Lumpur : Ministry of Health Malaysia ; Retrieved 16 September 2019 From http://iku.moh.gov.my/images/IKU/Document/REPORT/nhmsreport2015vol2.pdf

Ng M, Fleming T, Robinson M, Thomson B, Graetz N, Margono C, Mullany EC, Biryukov S, Abbafati C, Abera SF, Abraham JP, Abu-Rmeileh NM, Achoki T, AlBuhairan FS, Alemu ZA, Alfonso R, Ali MK, Ali R, Guzman NA, Ammar W, Anwari P, Banerjee A, Barquera S, Basu S, Bennett DA, Bhutta Z, Blore J, Cabral N, Nonato IC, Chang JC, Chowdhury R, Courville KJ, Criqui MH, Cundiff DK, Dabhadkar KC, Dandona L, Davis A, Dayama A, Dharmaratne SD, Ding EL, Durrani AM, Esteghamati A, Farzadfar F, Fay DF, Feigin VL, Flaxman A, Forouzanfar MH, Goto A, Green MA, Gupta R, Hafezi-Nejad N, Hankey GJ, Harewood HC, Havmoeller R, Hay S, Hernandez L, Husseini A, Idrisov BT, Ikeda N, Islami F, Jahangir E, Jassal SK, Jee SH, Jeffreys M, Jonas JB, Kabagambe EK, Khalifa SE, Kengne AP, Khader YS, Khang YH, Kim D, Kimokoti RW, Kinge JM, Kokubo Y, Kosen S, Kwan G, Lai T, Leinsalu M, Li Y, Liang X, Liu S, Logroscino G, Lotufo PA, Lu Y, Ma J, Mainoo NK, Mensah GA, Merriman TR, Mokdad AH, Moschandreas J, Naghavi M, Naheed A, Nand D, Narayan KM, Nelson EL, Neuhouser ML, Nisar MI, Ohkubo T, Oti SO, Pedroza A, Prabhakaran D, Roy N, Sampson U, Seo H, Sepanlou SG, Shibuya K, Shiri R, Shiue I, Singh GM, Singh JA, Skirbekk V, Stapelberg NJ, Sturua L, Sykes BL, Tobias M, Tran BX, Trasande L, Toyoshima H, van de Vijver S, Vasankari TJ, Veerman JL, Velasquez- Melendez G, Vlassov VV, Vollset SE, Vos T, Wang C, Wang X, Weiderpass E, Werdecker A, Wright JL, Yang YC, Yatsuya H, Yoon J, Yoon SJ, Zhao Y, Zhou M, Zhu S, Lopez AD, Murray CJ, Gakidou E. (2014) . Global, regional, and national prevalence of overweight and obesity in children and adults during 1980-2013: a systematic analysis for the Global Burden of Disease Study . Lancet, 384, 766-781. 
Russell, H. A., Rufus, C., Fogarty, C.T., Fiscella, K., Carroll, J. (2013). "You need a support. When you don't have that chocolate looks real good". Barriers to and facilitators of behavioural changes among participants of a Healthy Living Programme. Family Practice, 30, 452-458.

Sheperis, C.J., Young, J.S., \& Daniels, M.H. (2010).Counseling research : Quantitative, qualitative and mixed methods. Pearson Higher Education.

Spahn, J.M, Reeves, R.S., Keim, K.S, Laquatra, I, Kellogg, M., Jortberg, B., Clark, N.A. (2010). State of the evidence regarding behavior change theories and strategies in nutrition counseling to facilitate health and food behavior change. Journal of the American Dietetic Association, 110, 879-891.

Stokols, D. (1996). Translating Social Ecological Theory into Guidelines for Community Health Promotion. Journal of American Health Promotion, 10 (4), 282-298.

Tan, S. T., Mohd-Sidik, S., Rampal, L., Ibrahim, N., \& Tan, K. A. (2019) Are Malaysians Getting Fatter and Rounder?: An Updated Systematic Review (2009-2015). Malaysian Journal of Medicine and Health Sciences, 15 (1): 63-77

Tremmel, M., Gerdtham, U. G., Nilsson, P. M., \& Saha, S. (2017). Economic burden of obesity: a systematic literature review. International journal of environmental research and public health, 14(4), 435.

World Health Organization (2014). Non-communicable Diseases (NCD) Country profile. 